\title{
Diabetic neuropathy in NIDDM
}

\author{
J.D. Ward \\ D iabetes R esearch U nit, R oyal H allamshire H ospital, Sheffield, U K
}

Very little information is available as to major differences in the neuropathy seen in insulin-dependent (IDDM) and non-insulin-dependent (NIDDM) diabetes mellitus but the possibility that there is a difference might lead to an increased understanding of pathogenetic mechanisms in both types of neuropathy. U nfortunately, when workers studying a condition cannot agree on a definition relating either to clinical features or to physiological measurement, it is unlikely that important differences will be detected in the neurological status of IDDM or NIDDM. In this review attention will be paid to problems of definition and their solution, the prevalence of diabetic neuropathy in NIDDM compared to IDDM with comments about observed clinical features and histological differences.

Definition and recognition of diabetic neuropathy

It seems likely that three different definitions of diabetic neuropathy are required.

1. For large epidemiological studies.

2. For detailed clinical therapeutic and natural history studies.

3. For the practising physician faced with the task of identifying those subjects with a neuropathic problem or likely to develop one.

A recent consensus statement of the Peripheral Nerve Society defines diabetic neuropathy as "A symmetrical sensorimotor polyneuropathy predominantly affecting the distal aspects of the lower limbs" [1]. The purpose of such a definition is to establish a norm for

Corresponding author: Prof. J.D.Ward, Diabetes R esearch U nit, R oyal Hallamshire H ospital, Sheffield SIO 2J F, UK A bbreviations: IDDM, Insulin-dependent diabetes mellitus; NID D M , non-insulin-dependent diabetes mellitus. the majority of patients to whom the label diabetic neuropathy should be attached and to exclude variable clinical syndromes with very unpredictable features and natural history which would interfere with observations in a longitudinal clinical trial or study. H ence, sensory symptoms and deficits and a variable degree of autonomic dysfunction are the prominent features of this neuropathy and the consensus stresses that muscle weakness is infrequent.

This definition specifically excludes interesting clinical syndromes which occur in both IDDM and NIDDM : proximal diabetic neuropathy or diabetic amyotrophy, visceral autonomic neuropathy, multiple mononeuropathies, truncal radiculopathy, cranial neuropathy and any diabetic mononeuropathy and insulin neuritis. However, these less common clinical syndromes represent approximately $10 \%$ of patients seen in clinical practice with diabetic neuropathy and it might be that an understanding of the process leading to the clinical situation would lead to more understanding of the pathogenesis. A very detailed method of staging and defining neuropathy has been proposed by $D$ yck et al. [2] H owever, this process is very timeconsuming and costly and many workerswill be forced to look for ways to simplify the definitions for clinical studies. Such a standardised approach has been described by Feldman et al. [3] and could well form the basis of an agreed protocol on the diagnosis and staging, thus achieving agreement across studies when comparisons of patients are made [3]. U sing this approach a score is produced from careful measurement of symptoms and basic physical signs of light touch, tuning fork for vibration sensation, foot inspection and muscle strength assessment coupled with measures of conduction velocity, vibration perception and autonomic function tests. Four categories of neuropathy can therefore be described from nil to severe which has a score of 46 points. I important differences are to be observed in the neuropathy of NIDDM then 
the type of studies so far performed should be repeated using this generally agreed protocol.

P revalence studies of diabetic peripheral neuropathy

The wide variety of definitions and descriptions of neuropathy reported does not allow accurate conclusions to be drawn, but the overall impression is that there does not seem to be a major difference in the occurrence or features of nerve damage between the two conditions. In this review no special comment will be made about the varying definitions used in individual studies.

In a hospital-based study of 6487 subjects neuropathy was found to be present in $32.1 \%$ of those with NIDDM compared to $22.7 \%$ with IDDM [4]. A reasonable difference, but since patients in this study showed an increased prevalence with age and duration of diabetes and that these factors have been shown to be major determinants of neuropathy in all reported studies, it is extremely difficult to be certain as to the duration of diabetes in NIDDM and perhaps the differences are neither surprising nor particularly revealing. O ther clinic-based studies have shown no significant difference between the occurrence in both diseases [5].

In a community-based study of 1150 diabetic subjects compared to 480 normal non-diabetic subjects, $16.3 \%$ of those with diabetes were found to have neuropathy (compared to $2.9 \%$ in the normal population). N europathy was diagnosed in $17.2 \%$ of those with NIDDM and $12.7 \%$ for IDDM , again perhaps for the same reasons relating to the duration of the disease [6].

Ziegler and co-workers [7] have provided us with data in a large number of subjects using an acceptable definition of neuropathy not far from the Feldman protocol. Patients either had no symptoms (130 IDDM patients, 45 NIDDM patients), or had symptomatic neuropathy (65 ID D M patients, 91 NIDDM patients). Tests of temperature discrimination, pain threshold, heat and cold, vibration perception and conduction velocity were all more abnormal in those with NIDDM ( $p \geq 0.05)$, a small difference on which to base fundamental differences of pathogenesis and again possibly relating to the longer disease duration in the NIDDM subjects. In a smaller study no difference in clinical features could be detected between 52 subjects with IDD M compared to 36 subjects with NIDDM and there was no difference in conduction velocity [8].

Two other studies have confirmed a trend toward abnormalities of peripheral nerve function tests in NIDDM . In 1083 patients with an abnormal pin-prick test neuropathy was present in $16.8 \%$ of NIDDM compared to $8.8 \%$ of IDDM subjects [9]. The influence of blood glucose control is suggested in a study where in a normal non-diabetic population of 488 subjects, $3.9 \%$ were assessed as having some peripheral neurological deficit. This compared to $11.2 \%$ in 89 subjects with impaired glucose tolerance and $25 \%$ in 279 subjects with NIDDM [10].

Certainly significant neuropathy is not described at the onset of IDD M whereas significant degrees of nerve dysfunction and some symptomatology is seen in NIDDM at diagnosis, attesting to the obvious and unmeasurable duration of the disease and its effects on nerve function $[11,12]$.

P revalence studies in autonomic neuropathic dysfunction

Dysfunction of the autonomic system as demonstrated by the wide variety of functional tests has also been studied in the two conditions with some differences. In 0 xford 202 subjects with NIDDM were shown to have $15.8 \%$ of abnormalities compared to $20.9 \%$ in 43 subjects with ID D M [13]. A large multicentre German study using two measures of abnormality of cardiac autonomic function showed $22.1 \%$ of 524 subjects with NIDDM to be abnormal compared to $16.8 \%$ of those with IDDM [14].

A ttempts have also been made to measure antibodies to neurological tissue when studying the abnormalities of autonomic function in the two conditions. In one such study of 74 patients with IDDM compared to 38 patients with NIDDM, sympathetic nerve antibodies were decreased in the former group with autonomic neuropathy, while in subjects with NID D M, parasympathetic nerve antibodies were related to significant alteration in abnormalities of parasympathetic tests [15].

\section{Pathological features}

Very little data is available as to the neuropathological features of nerve damage in both forms of diabetes. Comparing 11 patients with IDDM and 17 with NIDDM Sima et al. [16] suggested that the pattern of nerve fibre loss seemed similar although in NIDDM wallerian degeneration was more prominent with a more obviously patchy loss of fibres, perhaps supporting a microvascular aetiology.

\section{Clinical features}

R eview of standard textbooks of clinical diabetes [17, 18] does not give an impression of any major difference in clinical features. Certainly clinical autonomic neuropathy is very unusual in NIDDM. The unusual but well-recognised syndrome of insulin neuritis 
(severe painful neuropathy developing 6 weeks or so after control of blood glucose, usually with insulin) occurs mainly in NIDDM subjects. The importance of the different clinical syndromes which might occur in the two conditions is that their recognition could lead to insights into pathogenetic mechanisms. The occurrence of severe autonomic disease in IDDM suggests the potential for immunological involvement in the pathology of this condition. The development of insulin neuritis in NIDDM subjects suggests that, for this breakdown to occur, nerves need to be already significantly compromised or damaged and that gross microvascular features present in the condition relate to pre-existing vascular vulnerability.

On balance therefore, there does not seem to be a major difference in the response of the peripheral nervous system to the metabolic insults received in both forms of diabetes. This would suggest similar pathways of pathogenesis. Of importance is that when suitable therapy is available for the prevention or treatment of nerve damage in diabetes, such treatment may be applied to those with both diseases.

\section{References}

1. Diabetic polyneuropathy in controlled clinical trials(1995) Consensus report of the peripheral nerve society. A $\mathrm{nn} \mathrm{N} \mathrm{e-}$ urol 38: 478-482

2. Dyck PJ (1988) D etection, characterisation and staging of polyneuropathy assessed in diabetics. Muscle Nerve 11: 21-32

3. Feldman E L, Stevens MJ, Thomas PK, B rown M B, Canal D, Greene DA (1994) A practical two step quantitative clinical and electrophysiological assessment for the diagnosis and staging of diabetic neuropathy. Diabetes Care 17: 1281-1289

4. Young $M J$, Boulton $A J M, M$ acL eod A F, Williams DRR, Sonksen PH (1993) A multicentre study of the prevalence of diabetic peripheral neuropathy in the U nited K ingdom hospital clinic population. D iabetologia 36: 150-154

5. Newrick PG, Boulton A J M , Ward J D (1986) The distribution of diabetic neuropathy in a British clinic population. D iabetes R es Clin Pract 2: 263-268
6. Walters DP, G atling W, Mullee MA, Hill RD (1992) The prevalence of diabetic distal sensory neuropathy in an E nglish community. D iabet $M$ ed 9: 349-353

7. Ziegler D, M ühlen H, G ries FA (1992) Neurophysiological tests in type 1 (insulin-dependent) and type 2 (non-insulindependent) diabetic patients with sub-clinical and symptomatic neuropathy. D iabetologia 35: 1099-1100

8. $\mathrm{H}$ endriksen $\mathrm{PH}$, O ey $\mathrm{PL}$, Wieneke $\mathrm{GH}$, Bravenboer $\mathrm{B}$, B anga J D (1992) Subclinical diabetic neuropathy: similarities between electrophysiological results in patients with type 1 (insulin-dependent) and type 2 (non-insulin-dependent) diabetes mellitus. D iabetologia 35: 690-695

9. Knuiman MW, Welborn TA, M CCann VJ (1986) Prevalence of diabetic complications in relation to risk factors. D iabetes 35: 1332-1339

10. Franklin G M , K ahn L B, B axter J (1990) Sensory neuropathy in non-insulin-dependent diabetes mellitus. The San L ouis Valley D iabetes Study. A m J E pidemiol 131: 633-643

11. Lehtinen J M, U usitupa M, Siitonen O, Pyorala K (1989) Prevalence of neuropathy in newly diagnosed NIDDM and non-diabetic control subjects. D iabetes 38: 1307-1313

12. G regory R, Tattersall RB, A llison SP (1994) Peripheral neuropathy as a presenting feature of type 2 diabetes: a case control study. D iabet M ed 11: 407-409

13. N eil HA W, Thompson A V, John S (1989) Diabetic autonomic neuropathy: the prevalence of impaired heart rate variability in a geographically defined population. Diabet Med 6: 20-24

14. Ziegler D, Gries FA, Spuler M, Lessmann F (1992) Diabetic cardiovascular autonomic neuropathy multi-centre study group: epidemiology of diabetic neuropathy. J Diab Comp 6: 49-57

15. Sundkvist G, Lind P, B ergstrom B, Lilja B, R abinowe SI (1991) A utonomic nerve antibodies in autonomic function in type 1 and type 2 diabetic patients. J Intern M ed 229: 505-510

16. Sima A A F, Nathaniel V, B ril V, McE wane TA J, Greene DA (1988) Histopathological heterogeneity of neuropathy in insulin dependent and non-insulin dependent diabetes and demonstration of axoglyol dysjunction in human diabetic neuropathy. J Clin Invest 81: 349-364

17. Thomas PK, Ward J D, Watkins PJ (1982) Chapter; D iabetic N europathy. In: K een H, Jarrett J (eds) Complications of diabetes. E dward A rnold, L ondon, pp 109-136

18. Ward J D (1994) Chapter 62; Clinical aspects of diabetic somatic neuropathy. In: Pickup J C, W illiams G (eds) Chronic complications of diabetes. Blackwell Scientific, London, pp 623-634 